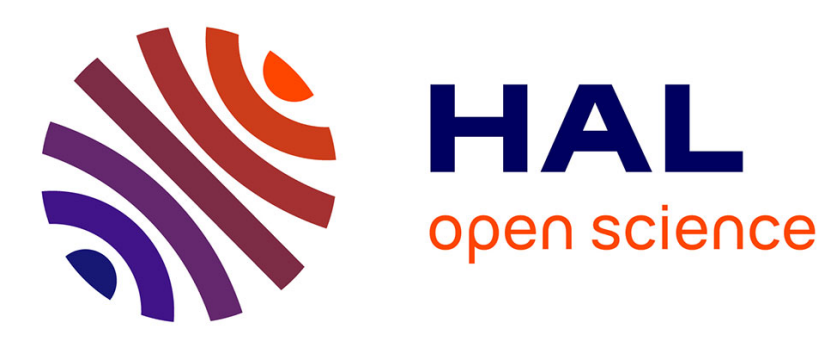

\title{
Cost Evaluation for Hybrid Inclusions: A Lyapunov Approach
}

Francesco Ferrante, Ricardo Sanfelice

\section{To cite this version:}

Francesco Ferrante, Ricardo Sanfelice. Cost Evaluation for Hybrid Inclusions: A Lyapunov Approach. 57th IEEE Conference on Decision and Control, Dec 2018, Miami, FL, United States. pp.855-860, 10.1109/CDC.2018.8619155 . hal-01850393v2

\section{HAL Id: hal-01850393 \\ https://hal.science/hal-01850393v2}

Submitted on 2 Jan 2021 (v2), last revised 23 Jan 2021 (v3)

HAL is a multi-disciplinary open access archive for the deposit and dissemination of scientific research documents, whether they are published or not. The documents may come from teaching and research institutions in France or abroad, or from public or private research centers.
L'archive ouverte pluridisciplinaire HAL, est destinée au dépôt et à la diffusion de documents scientifiques de niveau recherche, publiés ou non, émanant des établissements d'enseignement et de recherche français ou étrangers, des laboratoires publics ou privés. 


\title{
Cost Evaluation for Hybrid Inclusions: A Lyapunov Approach
}

\author{
Francesco Ferrante and Ricardo G. Sanfelice
}

\begin{abstract}
Cost evaluation problems for hybrid inclusions are studied. Sufficient conditions, in the form of Lyapunov-like inequalities, are provided to derive an upper bound on the cost associated with the solution to a hybrid inclusion with respect to a hybrid cost functional. Under additional sufficient conditions, we determine the cost exactly without computing solutions. Constructive results are proposed to solve cost evaluation problems in some relevant applications. Numerical examples are presented.
\end{abstract}

\section{INTRODUCTION}

Hybrid dynamical systems are dynamical systems whose evolution is characterized by the interplay of continuoustime dynamics and instantaneous changes. Due to the large number of applications in which hybrid dynamical systems can be used as a modeling paradigm, such a topic has gained an increasing interest over the last two decades. Research efforts in hybrid dynamical systems brought to life numerous tools for modeling, analysis, and design of hybrid systems; see [9], [15], [17], [24], [25]. In particular, in [9] a general framework for hybrid systems is established. The key feature of the framework in [9] consists of modeling hybrid dynamical systems via hybrid inclusions. Such a modeling approach allows one to deal with robustness aspects in hybrid systems in an elegant and unified way.

More precisely, a hybrid inclusion is formally written as

$$
\left\{\begin{array}{cccc}
\dot{x} & \in F(x) & & x \in C \\
x^{+} & \in G(x) & x \in D
\end{array}\right.
$$

where $x$ is the state, and $F$ and $G$ are set-valued mappings describing the dynamics of the system. The notation $\dot{x}$ represents the time derivative of the state, while $x^{+}$represents the value of the state after an instantaneous jumps. With these definitions, the above writing suggests that the state $x$ evolves according to the differential inclusion $\dot{x} \in F(x)$ while in $C$, and its value changes according to the difference inclusion $x^{+} \in G(x)$ when $x$ is in $D$.

Optimality aspects in hybrid systems have seen a growing interest in the community. First results on optimal control of hybrid systems can be traced back to the 90's in the work of Sussmann [23], later followed by [4], [22], where maximum principles for some class of hybrid and switched systems

Francesco Ferrante is with Univ. Grenoble Alpes, CNRS, GIPSA-lab, F-38000 Grenoble, France. Ricardo G. Sanfelice is with the Computer Engineering Department, University of California, Santa Cruz, CA 95064, USA. Email: francesco.ferrante@gipsa-lab.fr, ricardo@ucsc.edu.

This research has been partially supported by the National Science Foundation under CAREER Grant no. ECS-1450484 and Grant no. CNS1544396, and by the Air Force Office of Scientific Research under Grant no. FA9550-16-1-0015. This work has been done when the first author was with the Computer Engineering Department, University of California Santa Cruz. are formulated. More recently, several research directions concerning optimality in hybrid systems have been explored within the framework of hybrid inclusions in [9]. In [7] connections between pointwise stability and optimal control of hybrid systems are investigated. In [20], linear-quadratic optimal control for hybrid systems with linear dynamics and periodic jumps is studied. Cost evaluation problems play a central role in guaranteed cost control [5], so the solution to such a problem can be used to develop sub-optimal control design tools. For the class of linear-quadratic problems, i.e., linear dynamics and quadratic costs, closed form expressions of the cost value can be obtained by relying on the solution to a differential Riccati equation; see, e.g., [13, Chapter 6.1.3]. Unfortunately, as pointed out in [2], this technique cannot be applied when the cost is nonquadratic and does not extend to nonlinear systems. To overcome this problem, the idea proposed in [2] consists of establishing a connection between the cost functional and a Lyapunov-like inequality.

In this paper we take a first step towards the development of connections between Lyapunov theory and optimal control for hybrid systems modeled by hybrid inclusions. In particular, motivated by the general ideas originally presented in [2] for continuous-time systems and later extended in [6] to constrained difference inclusions, the problem we address consists of evaluating the cost associated to the solutions to a hybrid inclusion with respect to a given cost functional. Building from Lyapunov theory for hybrid systems in [9], we extend the results in [2] to hybrid inclusions. More precisely, the contributions in this paper are as follows. First, we provide sufficient conditions for cost evaluation for hybrid inclusions. In particular, we show that under some Lyapunov-like conditions, the cost associated to the solution to a hybrid inclusion, from a given initial condition, with respect to a hybrid cost functional can be upper bounded by a function of the initial condition. As a second step, we show that by strengthening some assumptions, the cost associated to the hybrid inclusion can be perfectly determined via the proposed conditions. Unlike previous results, uniqueness of solutions is nowhere assumed in the paper and when multiple solutions exist from a given initial condition, we take the supremum of the cost over all possible solutions. Finally, it is shown that in some particular applications, the proposed methodology leads to constructive conditions that can be easily used to solve the considered cost evaluation problem.

The remainder of this paper is structured as follows. Section $\amalg-\mathrm{A}$ presents some preliminaries on hybrid inclusions. Section $\amalg-\mathrm{B}$ and Section $\amalg-\mathrm{C}$ present our main results concerning the considered cost evaluation problems. Section III shows how our results can be specialized to deal with some 
relevant applications and presents some numerical examples. Due to space constraints, proofs of the main results are omitted and will be published elsewhere.

Notation: The symbol $\mathbb{N}_{>0}$ denotes the set of strictly positive integers, $\mathbb{N}=\mathbb{N}_{>0} \cup\{0\}, \mathbb{R}_{\geq 0}$ represents the set of nonnegative real scalars, $\mathbb{S}^{n}$ denotes the set of real symmetric matrices of dimension $n$ and $\mathbb{S}_{+}^{n}$ denotes the set of real symmetric positive definite matrices of dimension $n$. In partitioned symmetric matrices, the symbol • stands for symmetric blocks. The matrix $\operatorname{diag}\left\{A_{1}, A_{2}, \ldots, A_{n}\right\}$ is the block-diagonal matrix having $A_{1}, A_{2}, \ldots, A_{n}$ as diagonal blocks. For a vector $x \in \mathbb{R}^{n},|x|$ denotes the Euclidean norm, while $x_{i}$ denotes its $i$-th entry, and $\mathbf{1}_{n}$ denotes the vector in $\mathbb{R}^{n}$ whose entries are equal to one. Given two vectors $x, y$, we denote $(x, y)=\left[\begin{array}{ll}x^{\top} & y^{\top}\end{array}\right]^{\top}$, where $x^{\top}$ denotes the transpose of $x$. Given $M \in \mathbb{S}^{n}$, we denote by $\mathcal{C}(M)$ the negative cone generated by $M$, i.e., $\mathcal{C}(M):=\left\{x \in \mathbb{R}^{n}: x^{\top} M x \leq 0\right\}$. Given a vector $x \in \mathbb{R}^{n}$ and a closed set $\mathcal{A}$, the distance of $x$ to $\mathcal{A}$ is defined as $|x|_{\mathcal{A}}=$ $\inf _{y \in \mathcal{A}}|x-y|$. Given a set $S$, we denote $\bar{S}$ the closure of $S$.

\section{Cost Evaluation FOR HybRid InClusions}

\section{A. Preliminaries on Hybrid Inclusions}

We consider hybrid inclusions with state $x \in \mathbb{R}^{n}$ of the form

$$
\mathcal{H}_{0}\left\{\begin{array}{cccc}
\dot{x} & \in & F(x) & \\
x^{+} & \in & G(x) & x \in D
\end{array}\right.
$$

In particular we denote, $F: \mathbb{R}^{n} \rightrightarrows \mathbb{R}^{n}$ as the flow map, $C \subset \mathbb{R}^{n}$ as the flow set, $G: \mathbb{R}^{n} \rightrightarrows \mathbb{R}^{n}$ as the jump map, and $D \subset \mathbb{R}^{n}$ as the jump set.

A set $E \subset \mathbb{R}_{\geq 0} \times \mathbb{N}$ is a hybrid time domain if it is the union of a finite or infinite sequence of intervals $\left[t_{j}, t_{j+1}\right] \times\{j\}$, with the last interval (if existent) of the form $\left[t_{j}, T\right)$ with $T$ finite or $T=\infty$. Given a hybrid time domain $E$, we denote $\sup _{j} E=\sup \left\{j \in \mathbb{N}: \exists t \in \mathbb{R}_{\geq 0}\right.$ s.t. $(t, j) \in$ $E\}$ and $\sup _{t} E=\sup \left\{t \in \mathbb{R}_{\geq 0}: \exists j \in \mathbb{N}_{0}\right.$ s.t. $\left.(t, j) \in E\right\}$. A hybrid signal $\phi$ is a function defined over a hybrid time domain. A hybrid signal $\phi: \operatorname{dom} \phi \rightarrow \mathbb{R}^{n}$ is a hybrid arc if $\phi(\cdot, j)$ is locally absolutely continuous for each $j$. In particular, we denote $\mathcal{X}$ the class of hybrid arcs with values in $\mathbb{R}^{n}$. Given a hybrid signal $u, \operatorname{dom}_{t} u:=\{t \in$ $\mathbb{R}_{\geq 0}: \exists j \in \mathbb{N}$ s.t. $\left.(t, j) \in \operatorname{dom} u\right\}$ and $\operatorname{dom}_{j} u:=\{j \in$ $\mathbb{N}_{0}: \exists t \in \mathbb{R}_{\geq 0}$ s.t. $\left.(t, j) \in \operatorname{dom} u\right\}$. Given a hybrid signal $u$, $s \in \operatorname{dom}_{t} u$, and $i \in \operatorname{dom}_{j} u, j(s)=\min \{j \in \mathbb{N}:(s, j) \in$ $\operatorname{dom} u\}$ and $t(i)=\min \left\{t \in \mathbb{R}_{\geq 0}:(t, i) \in \operatorname{dom} u\right\}$. A hybrid arc $\phi \in \mathcal{X}$ is a solution to $\mathcal{H}$ if $\phi$ satisfies the dynamics of $\mathcal{H}$; see [9] for more details on hybrid systems. A solution $\phi$ to $\mathcal{H}$ is maximal if it cannot be extended and is complete if dom $\phi$ is unbounded. Given a set $M$ and the hybrid inclusion $\mathcal{H}_{0}$, we denote by $\mathcal{S}_{\mathcal{H}_{0}}(M)$ the set of all maximal solutions $\phi$ to $\mathcal{H}_{0}$ with $\phi(0,0) \in M$. If no set $M$ is mentioned, $\mathcal{S}_{\mathcal{H}_{0}}$ is the set of all maximal solutions to $\mathcal{H}_{0}$.

\section{B. Upper bounds}

By following the general ideas proposed in [2], in this section we investigate how a Lyapunov-like function can be used to provide estimates of nonlinear cost functionals for a given hybrid inclusion.
For each initial condition $\xi \in \bar{C} \cup D$ to $\mathcal{H}_{0}$ in (1), consider the following cost:

$$
\begin{aligned}
\mathcal{J}(\xi)=\sup _{\phi \in \mathcal{S}_{\mathcal{H}_{0}}(\xi)} & \left(\int_{\operatorname{dom}_{t} \phi} q_{c}(\phi(s, j(s))) d s+\right. \\
& \left.\sum_{j=1}^{\operatorname{dom}_{j} \phi} q_{d}(\phi(t(j), j-1))\right)
\end{aligned}
$$

where $q_{c}: \bar{C} \rightarrow \mathbb{R}_{\geq 0}$ and $q_{d}: D \cup G(D) \rightarrow \mathbb{R}_{\geq 0}$.

Remark 1. Given $\phi \in \mathcal{S}_{\mathcal{H}_{0}}$, the definition of the cost (2) implies that, when $\operatorname{dom}_{j} \phi$ is bounded, the value of $\phi\left(t_{J}, \sup _{j} \operatorname{dom} \phi\right)$, with $t_{J}=\inf \{t \in$ $\left.\mathbb{R}_{\geq 0}:\left(t, \sup _{j} \operatorname{dom} \phi\right) \in \operatorname{dom} \phi\right\}$, does not contribute to the "jump cost". Obviously, this does not lead to any difference in the cost value when dom $\phi$ is unbounded in the $j$ direction. Such a formulation turns out to be convenient for our analysis.

Throughout the paper, given a solution $\phi$ to (1), we denote

$$
\begin{aligned}
\mathcal{J}_{\phi}:= & \lim _{\substack{(\tau, \iota) \in \operatorname{dom} \phi \\
(\tau, \iota) \rightarrow \sup \operatorname{dom} \phi}}\left(\int_{[0, \tau]} q_{c}(\phi(s, j(s))) d s+\right. \\
& \left.\sum_{j=1}^{\iota} q_{d}(\phi(t(j), j-1))\right)
\end{aligned}
$$

The following result can be established.

Proposition 1. Let $\xi \in \bar{C} \cup D, q_{c}: \bar{C} \rightarrow \mathbb{R}_{\geq 0}$, and $q_{d}: D \cup G(D) \rightarrow \mathbb{R}_{\geq 0}$. Let $V: \operatorname{dom} V \rightarrow \mathbb{R}$ with $\operatorname{dom} V \supset$ $\bar{C} \cup D \cup G(D)$ be continuously differentiable on an open set containing $\bar{C}$. Assume that

$$
\begin{array}{ll}
\sup _{f \in F(x)}\langle\nabla V(x), f\rangle+q_{c}(x) \leq 0 & \forall x \in C \\
\sup _{g \in G(x)} V(g)-V(x)+q_{d}(x) \leq 0 & \forall x \in D
\end{array}
$$

Let $\phi: \operatorname{dom} \phi \rightarrow \mathbb{R}^{n}$ be a solution to (1) from $\xi$. Assume that $(t, j) \mapsto V \circ \phi(t, j)$ is bounded. Then, $\mathcal{J}_{\phi}$ is a finite number and in particular

$$
\mathcal{J}_{\phi}+\limsup _{\substack{(\tau, \iota) \in \operatorname{dom} \phi \\(\tau, \iota) \rightarrow \sup \operatorname{som} \phi}} V(\phi(\tau, \iota)) \leq V(\xi)
$$

Sketch of the proof. Pick any solution $\phi$ to (1) from $\xi$ and observe that for each $(\tau, \iota) \in \operatorname{dom} \phi$

$$
\begin{aligned}
V(\phi(\tau, \iota))-V(\phi(0,0)) & =\int_{0}^{\tau} \frac{d}{d s} V(\phi(s, j(s))) d s+ \\
\sum_{j=1}^{\iota} & {[V(\phi(t(j), j))-V(\phi(t(j), j-1))] }
\end{aligned}
$$

By using (3a) and (3b), the latter implies

$$
V(\phi(\tau, \iota))-V(\phi(0,0)) \leq-\widetilde{\mathcal{J}}_{\phi}(\tau, \iota)
$$


where for each $(t, j) \in \operatorname{dom} \phi$

$$
\widetilde{\mathcal{J}}_{\phi}(t, j):=\int_{0}^{t} q_{c}(\phi(s, j(s))) d s+\sum_{i=1}^{j} q_{d}(\phi(t(i), i-1))
$$

Therefore, from (6) one gets

$$
V(\phi(t, j))+\tilde{\mathcal{J}}_{\phi}(t, j) \leq V(\xi) \quad \forall(t, j) \in \operatorname{dom} \phi
$$

Since by assumption $V \circ \phi$ is bounded, (7) implies that $(t, j) \mapsto \widetilde{J}_{\phi}(t, j)$ is bounded. Using nonnegativity of $q_{c}$ and $q_{d}$, one can conclude that

$$
\lim _{\substack{t, j) \in \operatorname{dom} \phi \\ t, j) \rightarrow \sup \operatorname{dom} \phi}} \tilde{\mathcal{J}}_{\phi}(t, j)=\mathcal{J}_{\phi}
$$

is a finite number. In particular, from (7) one has

$$
V(\xi) \geq \limsup _{\substack{(t, j) \in \operatorname{dom} \phi \\(t, j) \rightarrow \sup \operatorname{dom} \phi}}\left(V(\phi(t, j))+\widetilde{\mathcal{J}}_{\phi}(t, j)\right)=
$$

which gives (4), concluding the proof.

Proposition 1 by building on a suitable function $V$, provides an upper bound on the cost $\mathcal{J}_{\phi}$ that depends on the solution chosen from $\xi$. Next, by relying on further assumptions, for a given initial condition $\xi \in \bar{C} \cup D$, we provide an upper bound on the cost $\mathcal{J}(\xi)$ that is solution independent.

Corollary 1. Let $\mathcal{A} \subset \mathbb{R}^{n}$ be closed, $\xi \in \bar{C} \cup D, q_{c}: \bar{C} \rightarrow$ $\mathbb{R}_{\geq 0}$, and $q_{d}: D \cup G(D) \rightarrow \mathbb{R}_{\geq 0}$. Assume there exists a function $V: \operatorname{dom} V \rightarrow \mathbb{R}$, $\operatorname{dom} V \supset \bar{C} \cup D \cup G(D)$, that is continuously differentiable on an open set containing $\bar{C}$ and uniformly continuous on a neighborhood of $\mathcal{A}$ such that $V(\mathcal{A} \cap \operatorname{dom} V)=\{0\}$ and (3) holds. Furthermore, assume that each $\phi \in \mathcal{S}_{\mathcal{H}_{0}}(\xi)$ is such that

$$
\lim _{\substack{(t, j) \in \operatorname{dom} \phi \\ t, j) \rightarrow \sup \operatorname{dom} \phi}}|\phi(t, j)|_{\mathcal{A}}=0
$$

Then

$$
\mathcal{J}(\xi) \leq V(\xi)
$$

Remark 2. To get a solution independent upper bound on the cost, in the above result we assumed $V$ to be uniformly continuous on a neighborhood of $\mathcal{A}$. Indeed, since $V(\operatorname{dom} V \cap \mathcal{A})=\{0\}$, one can show that uniform continuity on a neighborhood of $\operatorname{dom} V \cap \mathcal{A}$ ensures that for any $(t, j) \mapsto \phi(t, j)$ such that $\phi$ approaches $\mathcal{A}, V \circ \phi$ approaches zero. Alternatively, building upon Proposition $\square$ to relax the uniform continuity requirement of $V$, one could assume that for each $\phi \in \mathcal{S}_{\mathcal{H}_{0}}(\xi)$ such that $|\phi|_{\mathcal{A}}$ approaches zero, one has

$$
\limsup _{\substack{(t, j) \in \operatorname{dom} \phi \\(t, j) \rightarrow \sup \operatorname{dom} \phi}} V(\phi(t, j))=0
$$

On the other hand, observe that when $\mathcal{A}$ is compact (which is often the case in applications), by the Heine-Cantor Theorem, continuity of $V$ on a neighborhood of $\mathcal{A}$ is enough.

Remark 3. Corollary 1 shows that when maximal solutions from $\xi$ converge to $\mathcal{A}$, then an upper bound on the cost $\mathcal{J}(\xi)$ (which is solution independent) is given by $V(\xi)$. On the one hand, when $q_{d}, q_{c}$, and $V$ are positive definite with respect to $\mathcal{A}$, (3) implies for any complete solution $\phi$, that $V \circ \phi$ approaches zero. On the other hand, for maximal solutions that are not complete, finite time convergence to $\mathcal{A}$ is needed. Conditions for finite-time convergence for hybrid systems are given in [12, Theorem 3.9].

\section{Exact cost evaluation}

In this section, our main objective is to obtain the exact value of the cost $\mathcal{J}(\xi)$ in (2) for a given initial condition $\xi$, without explicitly computing it. To that end, next, under further assumptions on the system data and a stronger condition than (3), we provide a way to determine the exact value of $\mathcal{J}(\xi)$ for a given initial condition $\xi \in \bar{C} \cup D$.

Corollary 2. Let $\mathcal{A} \subset \mathbb{R}^{n}$ be closed, $\xi \in \bar{C} \cup D, q_{c}: \bar{C} \rightarrow$ $\mathbb{R}_{\geq 0}, q_{d}: D \cup G(D) \rightarrow \mathbb{R}_{\geq 0}$, and $F(x)$ and $G(x)$ be compact, respectively, for each $x \in C$ and each $x \in D$. Assume that there exists a continuous function $V: \operatorname{dom} V \rightarrow \mathbb{R}$, $\operatorname{dom} V \supset \bar{C} \cup D \cup G(D)$, that is continuously differentiable on an open set containing $\bar{C}$ such that

$$
\begin{array}{ll}
\max _{f \in F(x)}\langle\nabla V(x), f\rangle+q_{c}(x)=0 & \forall x \in C \\
\max _{g \in G(x)} V(g)-V(x)+q_{d}(x)=0 & \forall x \in D
\end{array}
$$

Furthermore, assume that for any solution $\phi_{0}$ to (1) from $\xi$, $V \circ \phi_{0}$ is bounded. Pick any solution $\phi$ to the hybrid system

$$
\begin{array}{ll}
\dot{x} \in \underset{f \in F(x)}{\arg \max }\langle\nabla V(x), f\rangle & x \in C \\
x^{+} \in \underset{g \in G(x)}{\arg \max } V(g) & x \in D
\end{array}
$$

with $\phi(0)=\xi$ and let $\phi_{0}$ be any solution to (1) from $\xi$. Then, one has that $\mathcal{J}_{\phi_{0}}$ and $\mathcal{J}_{\phi}$ are finite and in particular

$$
\begin{aligned}
\mathcal{J}_{\phi_{0}}+\limsup _{\substack{(t, j) \in \operatorname{dom} \phi \\
(t, j) \rightarrow \sup \operatorname{dom} \phi}} V\left(\phi_{0}(t, j)\right) \leq \\
\mathcal{J}_{\phi}+\limsup _{\substack{(t, j) \in \operatorname{dom} \phi \\
(t, j) \rightarrow \sup \operatorname{dom} \phi}} V(\phi(t, j))=V(\xi)
\end{aligned}
$$

Moreover, if $V$ is nonnegative, uniformly continuous on neighborhood of $\mathcal{A}, V(\operatorname{dom} V \cap \mathcal{A})=\{0\}$, and there exists a maximal solution $\phi$ to 11 with $\phi(0,0)=\xi$ such that

$$
\lim _{\substack{(t, j) \in \operatorname{dom} \phi \\(t, j) \rightarrow \sup \operatorname{dom} \phi}}|\phi(t, j)|_{\mathcal{A}}=0
$$

then, one has

$$
\mathcal{J}(\xi)=V(\xi)
$$


The results given in this section extend previous results on cost evaluation for continuous-time nonlinear systems [2] and constrained difference inclusions [6] to hybrid inclusions. Similarly as in [2], [6], our results have strong connections to Lyapunov analysis. More specifically, the applicability of our results to specific examples requires the search of a suitable Lyapunov-like function, which is in general a challenging task. In the subsequent section, we show how our results can be be used in some relevant applications in a constructive fashion.

\section{APPLICATIONS AND EXAMPLES}

In this section, we specialize our results to two specific classes of cost evaluation problems. The first class of problems we analyze pertains to the case of hybrid systems with linear maps, conic flow and jump sets, and quadratic cost. This setting is relevant since hybrid systems with conic flow and jump sets arise in many different areas, such as reset control systems; see [18]. The second class of problems concerns the case of hybrid systems with linear maps, periodic jumps, and quadratic cost. Such a type of systems can be found in numerous applications such as sampleddata control [10] and has recently attracted the attention of researchers; see, e.g., [16], [20] just to mention a few. In particular, in [20] specific tools have been provided for the solution to quadratic optimal control problems for linear hybrid systems with periodic jump 4 .

\section{A. Linear-Quadratic Problems with Conic Flow and Jump} sets

Consider the following hybrid system 2

$$
\mathcal{H}_{C}\left\{\begin{aligned}
\dot{x} & =A_{c} x & & x \in C_{c}:=\mathcal{C}\left(M_{c}\right) \\
x^{+} & =A_{d} x & & x \in D_{c}:=\mathcal{C}\left(M_{d}\right)
\end{aligned}\right.
$$

where $M_{c}, M_{d} \in \mathbb{S}^{n}$ and $A_{c}, A_{d} \in \mathbb{R}^{n \times n}$. Then, we have the following result.

Proposition 2. Let $\mathcal{A}=\{0\}, \xi \in C \cup D, x \mapsto q_{c}(x):=$ $x^{\top} Q_{c} x$, and $x \mapsto q_{d}(x):=x^{\top} Q_{d} x$, where $Q_{c}, Q_{d} \in \mathbb{S}_{+}^{n}$. Assume that there exists $P \in \mathbb{S}_{+}^{n}, \tau_{1}, \tau_{2} \in \mathbb{R}_{>0}$ such that

$$
\begin{aligned}
& A_{c}^{\top} P+P A_{c}+Q_{c}-\tau_{1} M_{c} \leq 0 \\
& A_{d}^{\top} P A_{d}-P+Q_{d}-\tau_{2} M_{d} \leq 0
\end{aligned}
$$

Let $\phi \in \mathcal{S}_{\mathcal{H}_{C}}(\xi)$ and assume that $\phi$ is complete. Then

$$
\mathcal{J}_{\phi} \leq \xi^{\top} P \xi
$$

Moreover, if every $\phi \in \mathcal{S}_{\mathcal{H}_{C}}(\xi)$ is complete, one has

$$
\mathcal{J}(\xi) \leq \xi^{\top} P \xi
$$

Obviously the upper bound one gets is in general conservative. On the other hand, such a conservatism can be reduced

\footnotetext{
${ }^{1}$ Simulations of hybrid systems are performed in Matlab ${ }^{\circledR}$ via the Hybrid Equations (HyEQ) Toolbox [21].

${ }^{2}$ Given $M \in \mathbb{S}^{n}$, the symbol $\mathcal{C}(M)$ denotes the negative cone generated by $M$. In the published proceeding, $\mathcal{C}(M)$ is not defined and $\mathcal{C}^{+}(M)$ stands for the positive cone.
}

by suitably selecting the matrix $P$ in (14). In particular, $P$ can be selected to minimize a certain criterion. A possible choice to minimize $\xi^{\top} P \xi$ in all directions consists of picking $\lambda_{\max }(P)$ as a criterion; i.e., the induced 2-norm of $P$; see [3]. Pursuing this approach, $P$ can be taken as the solution to the following semidefinite program:

$$
\begin{aligned}
& \underset{P, \lambda, \tau_{1}, \tau_{2}}{\operatorname{minimize}} \lambda \\
& \lambda I-P \geq 0 \\
& A_{c}^{\top} P+P A_{c}+Q_{c}-\tau_{1} M_{c} \leq 0 \\
& A_{d}^{\top} P A_{d}-P+Q_{d}-\tau_{2} M_{d} \leq 0 \\
& \tau_{1}>0, \tau_{2}>0, \lambda>0, P \in \mathbb{S}_{+}^{n}
\end{aligned}
$$

An example within the setting considered in the above result is presented next.

Example 1. Consider the following data for the hybrid inclusion $\mathcal{H}_{C}$

$$
\begin{aligned}
& A_{c}=\left(\begin{array}{cc}
0 & 1 \\
-1 & 0
\end{array}\right), A_{d}=\exp \left(A_{c}-I\right), Q_{c}=Q_{d}=I \\
& M_{d}=-M_{c}=\left(\begin{array}{cc}
-1 & 0.5 \\
0.5 & 0
\end{array}\right)
\end{aligned}
$$

by [9, Proposition 6.10], it can be easily shown that the above definition of the data of $\mathcal{H}_{C}$ ensures that maximal solutions to $\mathcal{H}_{C}$ are complete. By solving (17) in Matlab ${ }^{\circledR}$ using the YALMIP package [14], combined with the solver Mosek [1], one gets

$$
P \approx\left(\begin{array}{cc}
1.526 & -0.5 \\
-0.5 & 2.526
\end{array}\right)
$$

Fig. 1 shows the unique maximal solution to $\mathcal{H}_{C}$ from $\xi=$ $(2,6)$. The solution converges to the origin and is Zeno. In Fig. 2 we report the evolution of the function

$$
\begin{aligned}
&(t, j) \mapsto \widetilde{\mathcal{J}}_{\phi}(t, j):=\int_{0}^{t} q_{c}(\phi(s, j(s))) d s+ \\
& \sum_{i=1}^{j} q_{d}(\phi(t(i), i-1))
\end{aligned}
$$

As expected, $\mathcal{J}(\zeta)=\lim _{t+j \rightarrow \infty} \widetilde{\mathcal{J}}_{\phi}(t, j)$ is upper bounded by $V(\xi)=\xi^{\top} P \xi$.

\section{B. Linear-Quadratic Problems with Periodic Jumps}

Consider the following hybrid system with state $x=$ $\left(x_{p}, \tau\right): \in \mathbb{R}^{n} \times[0, T]$

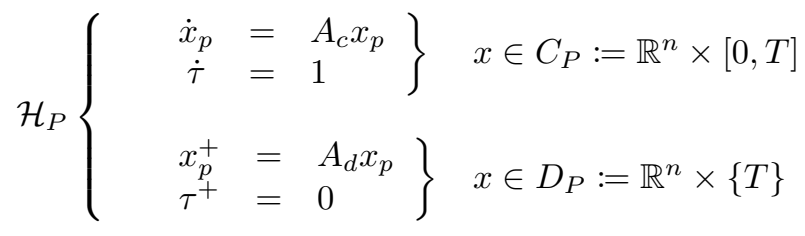

where $A_{c}, A_{d} \in \mathbb{R}^{n \times n}$, and $T>0$. We have the following result.

\footnotetext{
${ }^{3}$ Code at https://github.com/HybridSystemsLab/HybridCostLQConic
} 


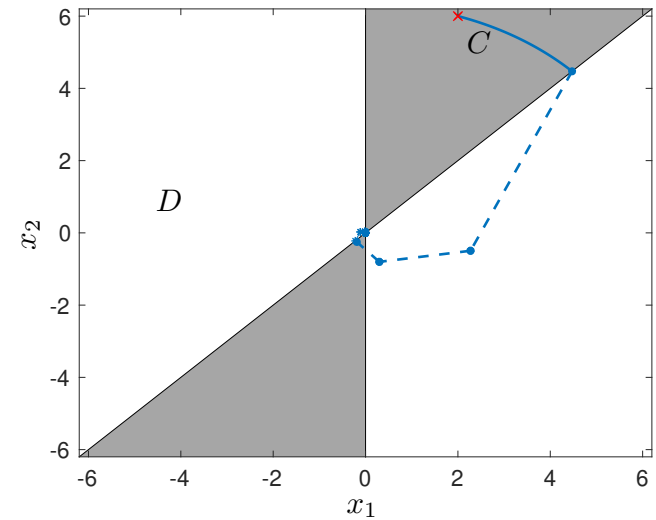

Fig. 1: The flow (grey) and jump (white) sets, and a trajectory from the initial condition $\xi=(2,6)$ (red $\times)$ for the system $\mathcal{H}_{C}$ in Example 1

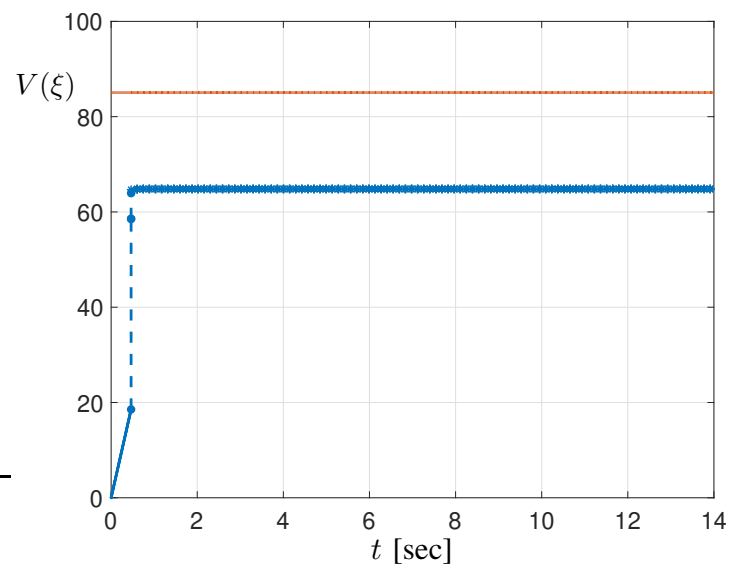

Fig. 2: The evolution of the function $\widetilde{\mathcal{J}}_{\phi}$ in Example 1

Proposition 3. Let $\mathcal{A}=\{0\} \times[0, T], \xi=\left(\xi_{p}, \xi_{\tau}\right) \in \mathbb{R}^{n} \times$ $[0, T], x \mapsto q_{c}(x):=x_{p}^{\top} Q_{c} x_{p}$, and $x \mapsto q_{d}(x):=x_{p}^{\top} Q_{d} x_{p}$, where $Q_{c}, Q_{d} \in \mathbb{S}_{+}^{n}$, and $x=\left(x_{p}, \tau\right)$. Furthermore, define

$$
\Psi(\tau):=e^{H(\tau-T)}=\left(\begin{array}{ll}
\psi_{1,1}(\tau) & \psi_{1,2}(\tau) \\
\psi_{2,1}(\tau) & \psi_{2,2}(\tau)
\end{array}\right) \quad \forall \tau \in[0, T]
$$

with

$$
H:=\left(\begin{array}{cc}
A_{c} & 0 \\
-Q_{c} & -A_{c}^{\top}
\end{array}\right)
$$

Assume that there exists $X \in \mathbb{S}_{+}^{n}$ such that

$$
X-A_{d}^{\top} e^{A_{c}^{\top} T} X e^{A_{c} T} A_{d}=A_{d}^{\top} \psi_{2,1}(0) e^{A_{c} T} A_{d}+Q_{d}
$$

and define for each $\tau \in[0, T]$

$$
P(\tau)=\left(\psi_{2,1}(\tau)+e^{-A_{c}^{\top}(\tau-T)} X\right) e^{-A_{c}(\tau-T)}
$$

Then

$$
\mathcal{J}(\xi)=\xi_{p}^{\top} P\left(\xi_{\tau}\right) \xi_{p}
$$

Sketch of the proof. First notice that due to the structure of $H$, it is straightforward to check that for each $\tau \in[0, T]$

$$
\Psi(\tau)=\left(\begin{array}{cc}
e^{A_{c}(\tau-T)} & 0 \\
\psi_{2,1}(\tau) & e^{A_{c}^{\top}(T-\tau)}
\end{array}\right)
$$

for some $\tau \mapsto \psi_{2,1}(\tau)$. Thanks to [19, Theorem 2.1] and due to the structure of $\Psi$ outlined in $[20$, it follows that $[0, T] \ni$ $\tau \mapsto P(\tau)$ in $18 \mathrm{~b}$, which is continuously differentiable on $(0, T)$, is the unique solution to the following final value problem:

$$
\begin{aligned}
& \frac{d}{d \tau} P(\tau)=-\operatorname{He}\left(A_{c}^{\top} P(\tau)\right)-Q_{c} \quad \forall \tau \in(0, T) \\
& P(T)=X
\end{aligned}
$$

Moreover, it can be proven that for each $\tau \in[0, T], P(\tau) \in$ $\mathbb{S}_{+}^{n}$. Define $C_{P} \ni x \mapsto V(x):=x_{p}^{\top} P(\tau) x_{p}$ and observe that, due to $P([0, T]) \subset \mathbb{S}_{+}^{n}, V$ is positive definite with respect to $\mathcal{A}$ on $C_{P}$. For all $x \in C_{P}$

$$
\left\langle\nabla V(x),\left(A_{c} x_{p}, 1\right)\right\rangle=x_{p}^{\top}\left(\operatorname{He}\left(P(\tau) A_{c}\right)+\frac{d}{d \tau} P(\tau)\right) x_{p}
$$

hence, thanks to 21a , the latter gives

$$
\left\langle\nabla V(x),\left(A_{c} x_{p}, 1\right)\right\rangle=-x_{p}^{\top} Q_{c} x_{p} \quad \forall x \in C_{P}
$$

which corresponds to 10a). Additionally, for all $x \in D_{P}$, one has

$$
V\left(\left(A_{d} x_{p}, 0\right)\right)-V(x)=x_{p}^{\top}\left(A_{d}^{\top} P(0) A_{d}-X\right) x_{p}
$$

By substituting the expression of $P(0)$ given by $18 \mathrm{~b}$ into (23) and by using (18a), one gets

$V\left(\left(A_{d} x_{p}, 0\right)\right)-V(x)=-x_{p}^{\top} Q x_{p} \quad x \in D_{P} \quad \forall x \in D_{P}$

which corresponds to $10 \mathrm{~b}$. At this stage, since $Q_{c}$ and $Q_{d}$ are positive definite and maximal solutions to $\mathcal{H}_{P}$ are complete, by [9, Theorem 3.18], (22)-(24) imply that maximal solutions to $\mathcal{H}_{P}$ converge to $\mathcal{A}$. Therefore, by invoking Proposition 11 (22) and (24) give (15) and the result is established.

Remark 4. It is worthwhile to observe that 21a) is a differential Riccati equation. The fact that our approach when specialized to the case of linear hybrid systems with periodic jumps leads to a differential Riccati equation is consistent with linear quadratic control theory. In particular, from linear quadratic control theory (see, e.g., [13, Chapter 6.1.3]), it is well-known that the solution $P:[0, T] \rightarrow \mathbb{S}_{+}^{n}$ to (21) is such that for any solution $\varphi$ to $\dot{x}=A_{c} x$ with $\varphi(0)=\xi_{p}$ and any $\theta \in[0, T]$, one has

$$
\xi_{p}^{\top} P(\theta) \xi_{p}=\int_{\theta}^{T} \varphi(s)^{\top} Q_{c} \varphi(s) d s+\varphi^{\top}(T) P(T) \varphi(T)
$$

Therefore, Proposition 3 establishes a direct relationship between the considered hybrid cost evaluation problem and a specific continuous-time finite-horizon linear quadratic

\footnotetext{
${ }^{4} \mathrm{~A}$ similar definition for the Lyapunov function appeared in [8, Example 26], though no cost evaluation is considered therein.
} 
cost evaluation problem. In particular, by comparing (25) with (19), Proposition 3 enables to conclude that, for any $\left(\xi_{p}, \xi_{\tau}\right) \in C_{P}$, the cost associated to the solutions to $\mathcal{H}_{P}$ with respect to the "hybrid" cost (2), with $q_{c}$ and $q_{d}$ are quadratic functions, coincides with

$$
\int_{\xi_{\tau}}^{T} \varphi(s)^{\top} Q_{c} \varphi(s) d s+\varphi^{\top}(T) P(T) \varphi(T)
$$

where for all $t \in(0, T), \dot{\varphi}(t)=A_{c} \varphi(t), \varphi(0)=\xi_{p}$, and the terminal-cost matrix $X$ is selected as in (18a).

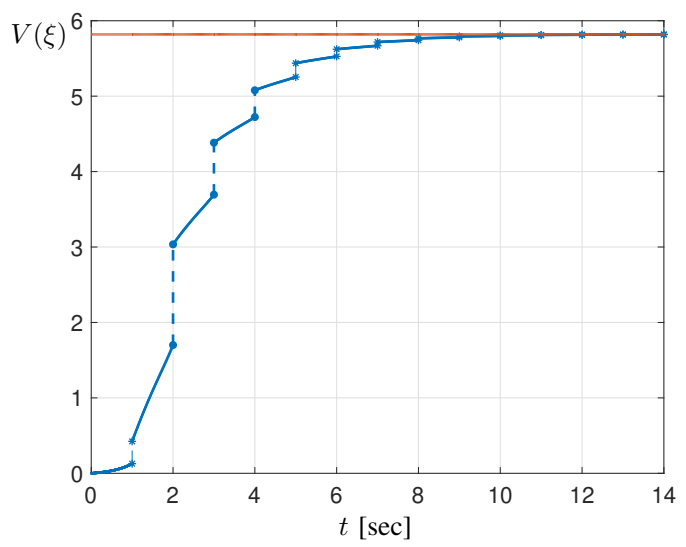

Fig. 3: The evolution of the function $\widetilde{\mathcal{J}}_{\phi}$ in Example 2

Example 2. Consider the following data for $\mathcal{H}_{P}$ :

$$
A_{c}=\left(\begin{array}{ll}
1 & 1 \\
0 & 0
\end{array}\right), A_{d}=\left(\begin{array}{cc}
1.0 & 0 \\
-2.0 & 0
\end{array}\right), T=1
$$

Solving (18a) in Matlab ${ }^{\circledR}$ yield $₫$ 苟 $X \approx\left(\begin{array}{cc}19.26 & 0 \\ \bullet & 1\end{array}\right)$. In Fig. 3 we report the evolution of the function

$$
(t, j) \mapsto \widetilde{\mathcal{J}}_{\phi}(t, j):=\int_{0}^{t} q_{c}(\phi(s, j(s))) d s+\sum_{i=1}^{j} q_{d}(\phi(t(i), i-1))
$$

with $\phi \in \mathcal{S}_{\mathcal{H}_{P}}(\xi)$ and $\xi=(0.2,0,0)$. As expected

$$
\mathcal{J}(\xi)=\lim _{t+j \rightarrow \infty} \widetilde{\mathcal{J}}_{\phi}(t, j)=V(\xi)
$$

\section{CONCLUSION}

In this paper we addressed cost evaluation problems for hybrid inclusions in the framework of [9]. The results are obtained by establishing a connection between a general hybrid cost functional and a Lyapunov like function. Sufficient conditions for exact cost evaluation are provided. Additionally, in some applications of relevant interest, our results have been specialized to get constructive tools for cost evaluation.

Future research directions include the extension of the proposed approach to hybrid optimal control. Moreover, the extension of the proposed approach to hybrid dynamical games in the spirit of [11] is part of our current research.

\section{REFERENCES}

[1] MOSEK ApS. The MOSEK optimization toolbox for MATLAB manual. Version 7.1 (Revision 28)., 2015.

[2] D. S. Bernstein. Nonquadratic cost and nonlinear feedback control. International Journal of Robust and Nonlinear Control, 3(3):211-229, 1993.

[3] S. Boyd, L. E. Ghaoui, E. Feron, and V. Balakrishnan. Linear Matrix Inequalities in System and Control Theory. Society for Industrial and Applied Mathematics, June 1997.

[4] P. E. Caines, F. H. Clarke, X. Liu, and R. B. Vinter. A maximum principle for hybrid optimal control problems with pathwise state constraints. In Proceedings of the 45th IEEE Conference on Decision and Control, pages 4821-4825. IEEE, 2006.

[5] S. H. Esfahani and I. R. Petersen. An LMI approach to the outputfeedback guaranteed cost control for uncertain time-delay systems. In Proceedings of the 37th IEEE Conference on Decision and Control, volume 2, pages 1358-1363. IEEE, 1998.

[6] F. Ferrante and R. G. Sanfelice. On the optimality of lyapunov-based feedback laws for constrained difference inclusions. In Proceedings of the American Control Conference, pages 3435-3440, 2018.

[7] R. Goebel. Optimal control for pointwise asymptotic stability in a hybrid control system. Automatica, 81:397-402, 2017.

[8] R. Goebel, R. G. Sanfelice, and A. R. Teel. Hybrid dynamical systems. Control Systems Magazine, 29(2):28-93, 2009.

[9] R. Goebel, R. G. Sanfelice, and A. R. Teel. Hybrid Dynamical Systems: Modeling, Stability, and Robustness. Princeton University Press, 2012.

[10] L. Hetel, C. Fiter, H. Omran, A. Seuret, E. Fridman, J.-P. Richard, and S. I. Niculescu. Recent developments on the stability of systems with aperiodic sampling: An overview. Automatica, 76:309-335, 2017.

[11] Andrea L'Afflitto. Differential games, continuous lyapunov functions, and stabilisation of non-linear dynamical systems. IET Control Theory \& Applications, 11(15):2486-2496, 2017.

[12] Y. Li and R. G. Sanfelice. Results on finite time stability for a class of hybrid systems. In Proceedings of the American Control Conference, pages 4263-4268, 2016.

[13] D. Liberzon. Calculus of variations and optimal control theory: A concise introduction. Princeton University Press, 2011.

[14] J. Lofberg. YALMIP: A toolbox for modeling and optimization in matlab. In Procceedings of the IEEE International Symposium on Computer Aided Control Systems Design, pages 284-289, 2004.

[15] J. Lygeros, Karl H. J., S. N. Simic, J. Zhang, and S. S. Sastry. Dynamical properties of hybrid automata. IEEE Transactions on automatic control, 48(1):2-17, 2003.

[16] L. Marconi and A. R. Teel. Internal model principle for linear systems with periodic state jumps. IEEE Transactions on Automatic Control, 58(11):2788-2802, 2013.

[17] A. N. Michel and B. Hu. Towards a stability theory of general hybrid dynamical systems. Automatica, 35(3):371-384, 1999.

[18] D. Nešić, L. Zaccarian, and A. R. Teel. Stability properties of reset systems. Automatica, 44(8):2019-2026, 2008.

[19] L. Ntogramatzidis and A. Ferrante. On the exact solution of the matrix riccati differential equation. In Proceedings of the 18th IFAC World Congress, pages 14556-14561, 2011.

[20] C. Possieri and A. R. Teel. LQ optimal control for a class of hybrid systems. In Proceeding of the IEEE 55th Conference on Decision and Control, pages 604-609, 2016.

[21] R. G. Sanfelice, D. Copp, and P. Nanez. A toolbox for simulation of hybrid systems in matlab/simulink: Hybrid equations (HyEQ) toolbox. In Proceedings of the 16th International Conference on Hybrid Systems: Computation and Control, pages 101-106. ACM, 2013.

[22] M. S. Shaikh and P. E. Caines. On the hybrid optimal control problem: theory and algorithms. IEEE Transactions on Automatic Control, 52(9):1587-1603, 2007.

[23] H. J. Sussmann. A maximum principle for hybrid optimal control problems. In Proceedings of the 38th IEEE Conference on Decision and Control, volume 1, pages 425-430, 1999.

[24] L. Tavernini. Differential automata and their discrete simulators. Nonlinear Analysis: Theory, Methods \& Applications, 11(6):665-683, 1987.

[25] A. J. Van Der Schaft and J. M. Schumacher. An introduction to hybrid dynamical systems, volume 251. Springer London, 2000. 